\title{
Studies with an enthesis index as a method of clinical assessment in ankylosing spondylitis
}

\author{
MICHELLE MANDER, JUDY M SIMPSON, ALISON MCLELLAN, \\ DAVID WALKER, JOHN A GOODACRE, AND W CARSON DICK
}

From the Departments of Rheumatology and Medical Statistics, University of Newcastle upon Tyne

SUMmARY The histopathological characteristic of ankylosing spondylitis (AS) is the presence of chronic enthesitis. Our aim was to develop a clinical measurement of the severity of tenderness over entheses. The scoring system was based on the patients' response to palpation over entheses easily accessible to examination. The enthesis index $(E I)$ correlated with pain $(r=0 \cdot 67, p<0 \cdot 01)$ and stiffness $(r=0.46, p<0.05)$ scores. A single, blind, crossover study was conducted to determine the sensitivity of the index to change in clinical state associated with non-steroidal antirheumatic drug therapy and to record the interobserver variability. The index showed significantly lower scores after one week's drug treatment $(p<0.05)$. The EI is a convenient, non-invasive measure of disease severity in patients with AS. Potential applications include the assessment of enthesitis in other polyarthritides and a means of distinguishing clinically between severity of enthesitis and synovitis in different types of polyarthritis.

AS is a chronic inflammatory disease, of which the characteristic histopathological feature is enthesitis. ${ }^{1}$ An enthesis is the site of attachment to bone of tendon, ligament, or joint capsule. ${ }^{2}$ Present methods of assessing disease activity are based on measurements of erythrocyte sedimentation rate (ESR), serum $C$ reactive protein concentration, and severity of pain and stiffness by graded or visual analogue scales (VAS). Recently, measurement of radioisotope uptake over sacroiliac joints has been used as an indicator of sacroiliitis. ${ }^{3}$ There are other methods of assessment in AS, for example, spondylometry, ${ }^{4}$ but there is no validated clinical method of assessing the severity of enthesitis in AS. We have developed such an index and determined its correlation with other measures of disease activity. We have also examined the ability of the index to detect change in clinical state in patients with AS. Our data show that the enthesis index is a useful clinical method for assessing disease activity in AS.

\section{Patients and methods}

The initial aim was to design an index encompassing

Accepted for publication 5 September 1986

Correspondence to Ms M Mander, University Department of Rheumatology, Royal Victoria Infirmary, Newcastle upon Tyne NE1 4LP. entheses easily accessible to clinical examination. This included entheses known to be involved in AS together with sites of possible involvement. The following sites were examined: the nuchal crests, the manubriosternal joint, the costochondral joints, the greater tuberosity and the medial and lateral epicondyles of the humerus, the iliac crests and the anterior superior iliac spines, the greater trochanter of the femur, the tibial tuberosities, the adductor tubercles, the medial and lateral condyles of the femur and tibia, the head of the fibula, the calcaneal insertions of the plantar fascia and the achilles tendons, the sacroiliac joints, the cervical, thoracic, and lumbar spinous processes, the ischial tuberosities, and the anterior posterior iliac spines.

A scoring system was developed based on the patients' response to firm palpation over these entheses. Some of the sites were scored individually, whereas others were scored as a group, the highest scoring site being recorded for the group as a whole. The sites which were grouped in this way were: the nuchal crests, the costochondral joints, the sacroiliac joints, and the cervical, thoracic, and lumbar spinous processes. The remaining sites were scored individually left and right.

We tested the index in a pilot study, the aim of which was to study the acceptability and convenience of the index and to determine whether it might be refined in any way. Six male outpatient 


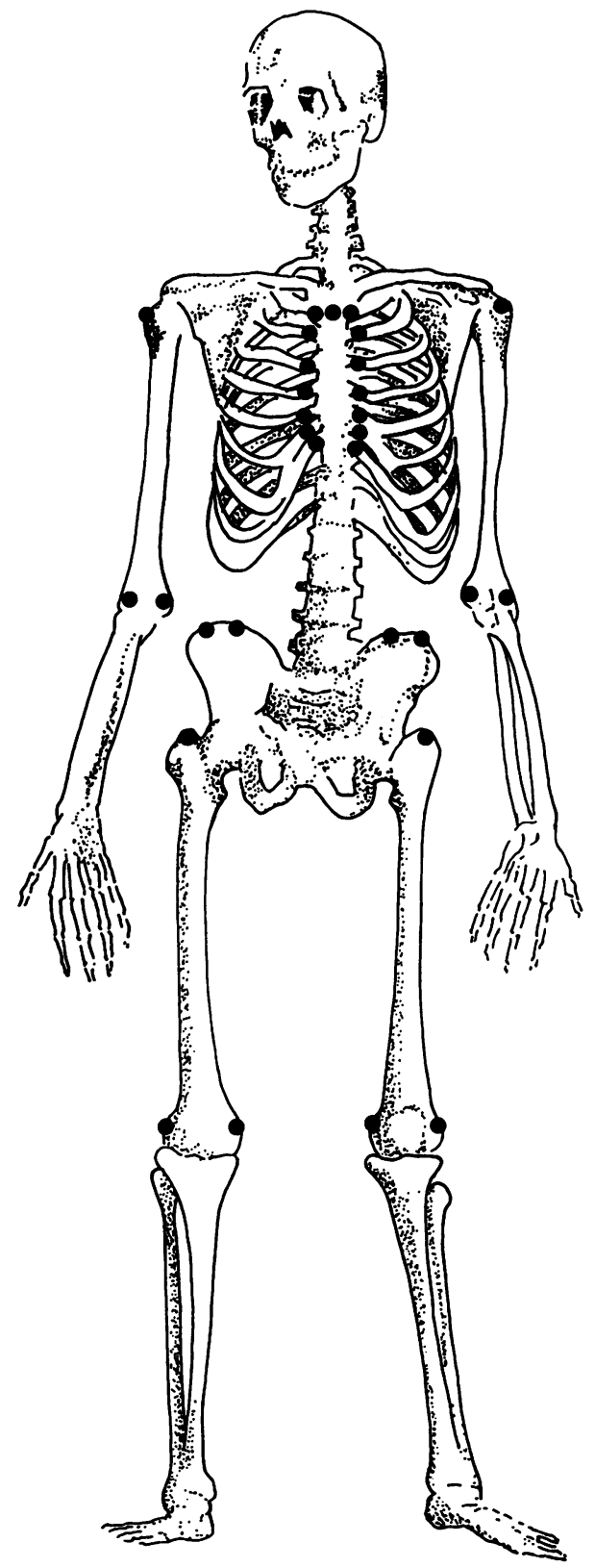

Fig. 1a

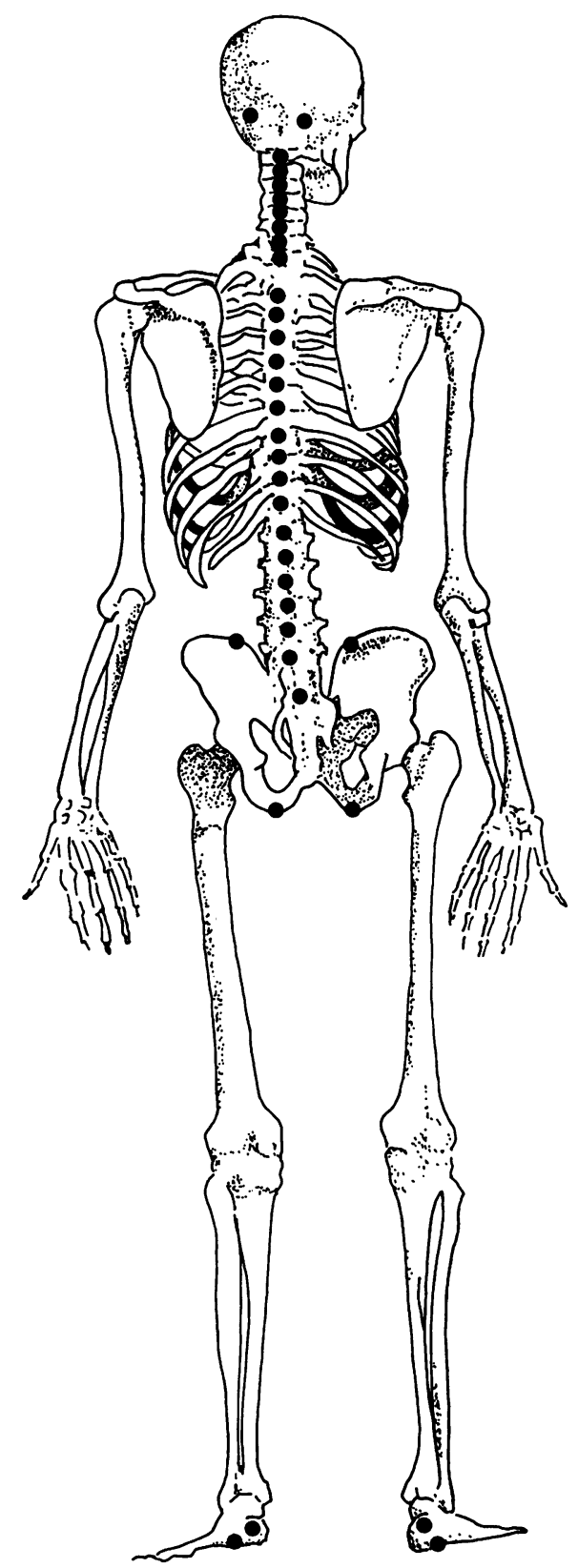

Fig. 1b

Fig. 1 Entheses examined with patient lying (a) supine, (b) prone.

volunteers, aged between 30 and 64 years, with disease duration ranging from three to 44 years, and with definite AS were assessed on two occasions one week apart. Each patient was assessed independently by the same three observers at the same time of day. On the first occasion the patients had discontinued antirheumatic drug therapy 48 hours previously, whereas on the second visit they had ${ }_{0}^{\circ}$ been taking standard non-steroidal antirheumatic $\mathbb{\mathbb { D }}$ drug therapy for one week.

Those sites which scored zero throughout this pilot study were eliminated, resulting in the final 
enthesis index (Figs 1a and b). We included the insertions of the achilles tendons and plantar fascia into the calcaneus, on the basis of published evidence of their involvement, ${ }^{5}$ though these sites were not commonly tender in our pilot study.

The scoring system was simplified by combining the two highest scoring categories, resulting in the following scheme: no pain $=0$; mild tenderness $=1$; moderate tenderness $=2$; wince or withdraw enthesis $=3$. These manoeuvres made no difference to the pattern of results obtained in this pilot study but produced a more concise index, the total possible score of which was 90 .

To improve discrimination between pressure and actual tenderness over the entheses a standard palpation was introduced at the beginning of each assessment. Pressure was applied over the anteriorsuperior aspect of the middle of the medial third of the clavicle so that the patient could compare the sensation at this point, arising from pressure alone, with that at the other sites palpated during the assessment. There is no enthesis at this aspect of the clavicle $^{6}$ and tenderness at this site is rare. In addition, the method of assessing the sacroiliac joints in the presence of severe flexion deformity of the lumbar spine was standardised. Patients who were unable to lie prone were assessed for sacroiliac tenderness lying on their side with force applied to the sacroiliac joint through the iliac crests.

The main study was then designed and conducted to investigate the efficacy of the index. The objectives of the study were: $(a)$ to determine the correlation between the enthesis index and other methods of assessing disease activity in patients with AS; $(b)$ to determine the interobserver error of the enthesis index; and $(c)$ to determine whether the enthesis index detects change in clinical state in patients with AS.

To pursue the first objective 19 patients were assessed on one occasion by one observer. The age range of the patients was 26-67 years (median age 45 years), disease duration ranged from three years to 44 years (median disease duration 20 years). Measurements were made of enthesis index and the severity of pain and stiffness using $10 \mathrm{~cm}$ horizontal visual analogue scales. The scales had the words no pain (or no stiffness) at the zero end of the scale and the words pain (or stiffness) could not be worse at the opposite end of the scale. The erythrocyte sedimentation rate was measured by the Westergren method. To pursue the second and third objectives 18 outpatients were assessed sequentially by three observers in a single, 'observer blind' study which used a crossover design. The age range of the patients was 14 to 72 years (median age 40 years), and the disease duration ranged from six months to
38 years (median disease duration 15 years). At the first visit patients were divided into groups: group A-two patients chose to continue receiving daily non-steroidal antirheumatic drug therapy throughout the study; group B-two patients chose not to receive non-steroidal antirheumatic therapy throughout the study; group $\mathrm{C}-14$ patients chose to participate in the crossover study. They were randomly allocated to two groups so that half took no drugs for the first week followed by daily non-steroidal antirheumatic drug therapy for the second week, while the other half did the reverse of this. The drugs were all preparations used in common clinical practice; optimal doses had been determined previously by the patients.

Weekly assessments over a three week period were made at the same time of day by the same three observers, who were unaware of the group to which the patient was allocated. Measurements were made of severity of enthesitis by enthesis index and of severity of pain and stiffness by visual analogue scales.

\section{STATISTICAL ANALYSIS}

Spearman's rank correlation coefficients were calculated using the data from 19 patients in the correlation study. To analyse the results of the crossover study (group C only) the scores for the enthesis index and VAS scores for pain and stiffness from the three observers were averaged; only the data for the last two visits were required for this analysis, and no significant difference between observers' scores had been found for these two visits. Distribution free statistical tests were used because the distribution of these variables is not known. Wilcoxon's signed rank test was used for the within patient comparison between measurements during non-steroidal antirheumatic drug treatment and in its absence (drug effect). Wilcoxon's rank sum test was used for the between patient comparisons: firstly, to test whether the drug effect depends on the sequence of the two treatments (period effect); and secondly, to test whether the average measurement for each patient depends on the sequence (sequence effect). To analyse interobserver variability (using groups A, B, and C) the results for each visit were analysed separately. As the measurements may not be normally distributed with homogeneous variance, distribution free statistical analysis was used. A Friedman two way analysis of variance by ranks was performed on the data from each visit.

\section{Results}

There were significant correlations between enthesis 
index and severity of pain $(\mathrm{p}<0.01)$ and stiffness $(p<0.05)$, but not between enthesis index and ESR (Table 1). There was no significant correlation between ESR and either pain or stiffness scores, showing that the ESR was a poor clinical indicator of disease activity in these patients.

The scores for enthesis index and severity of pain were significantly lower when the patients were

Table 1 Spearman's rank correlation coefficients

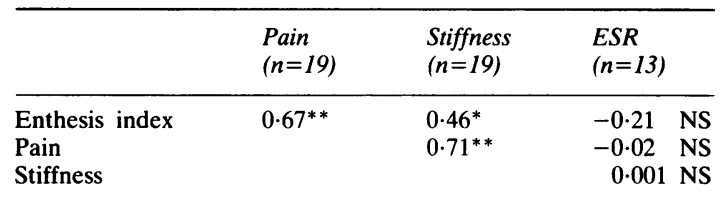

NS=not significant, $p>0.05$.

${ }^{*} \mathrm{p}<0.05$ (one sided); ${ }^{* *} \mathrm{p}<0.01$ (one sided).

Table 2 Results of significance tests of effects of drug, period, and sequence in crossover study

\begin{tabular}{llll}
\hline Measurement & Effect & & \\
\cline { 2 - 4 } & $\begin{array}{l}\text { Drug } \\
\text { Rank sum } \\
T_{14^{\dagger}}\end{array}$ & $\begin{array}{l}\text { Period } \\
\text { Rank sum } \\
T_{7.7}\end{array}$ & $\begin{array}{l}\text { Sequence } \\
\text { Rank sum } \\
T_{7.7}\end{array}$ \\
\hline Enthesis index & $21^{*}$ & $49 \mathrm{NS}$ & $41 \mathrm{NS}$ \\
Pain & $13^{*}$ & $52.5 \mathrm{NS}$ & $38 \mathrm{NS}$ \\
Stiffness & $21 \mathrm{NS}$ & $44.5 \mathrm{NS}$ & $39 \mathrm{NS}$ \\
\hline
\end{tabular}

$\mathrm{NS}=$ not significant, $\mathrm{p}>0.05$.

${ }^{*} \mathrm{p}<0.05$.

†For pain and stiffness scores $T_{13}$, i.c., 13 non-zero differences. taking a non-steroidal antirheumatic drug (Table 2). The median enthesis index scores were 10.5 with the $\Rightarrow$ drug and 13.7 without. For the pain scores the medians were 26.8 and 31.7 respectively. Stiffness $\bar{C}$ scores were not significantly affected by the drug; $\frac{\overline{\bar{O}}}{\overline{0}}$ median scores were 37.5 with the drug and $54.4 \frac{}{7}$ without. No significant period effect or sequence $\stackrel{\mathbb{Q}}{\varrho}$ effect was observed in any of the measurements.

On the first of the three visits there was significant $\vec{\partial}$ variability between observers in their measurements of the enthesis index but not in the patients' $\vec{\omega}$ assessment of pain or stiffness by visual analogue scales (Table 3).

These results show that the enthesis index is sensitive to change in clinical state associated with non-steroidal antirheumatic drug therapy in $\mathrm{AS}, \underset{\rightarrow}{\oplus}$ but, as would be expected with a subjective method 9 of assessment, there may be significant variation in 0 scoring between different observers using the index.

\section{Discussion}

Although the presence of an enthesitis is a recog- $\overrightarrow{\mathscr{Q}}$ nised feature of AS, there is no method of assessing. the severity of this component of the disease and 0 distinguishing it from severity of the synovitis that may also be present in the same or in different joints. There may be other causes of pain in AS which we have not attempted to measure in the present paper. We have designed and tested an enthesis index which shows significant correlation with other methods of assessing diseąse activity and which shows a significant reduction in score after the patient has taken optimal non-steroidal antirheumatic drug therapy for one week. The index is a method of assessment which is non-invasive, acceptable to all the patients in this study, and convenient

Table 3 Comparison of observers for enthesis index and visual analogue scale scores for pain and stiffness

\begin{tabular}{|c|c|c|c|c|c|c|c|c|c|}
\hline & \multicolumn{3}{|c|}{$\begin{array}{l}\text { Visit } 1 \\
\text { Observer }\end{array}$} & \multicolumn{3}{|c|}{$\begin{array}{l}\text { Visit } 2 \\
\text { Observer }\end{array}$} & \multicolumn{3}{|c|}{$\begin{array}{l}\text { Visit } 3 \\
\text { Observer }\end{array}$} \\
\hline & $I$ & 2 & 3 & 1 & 2 & 3 & 1 & 2 & -3 \\
\hline \multicolumn{10}{|l|}{ Enthesis index } \\
\hline Mean & 17 & 14 & 17 & 16 & 18 & 18 & 16 & 21 & 18 \\
\hline Mean rank & $2 \cdot 3$ & $1 \cdot 3$ & $2 \cdot 4^{* *}$ & $1 \cdot 6$ & $2 \cdot 3$ & $2 \cdot 1 \mathrm{NS}$ & $1 \cdot 8$ & $2 \cdot 5$ & $1.8 \mathrm{NS}$ \\
\hline \multicolumn{10}{|l|}{ Pain } \\
\hline Mean (mm) & 28 & 28 & 28 & 34 & 33 & 34 & 32 & 32 & 33 \\
\hline Mean rank & 1.9 & $2 \cdot 0$ & $2 \cdot 1 \mathrm{NS}$ & $1 \cdot 8$ & $2 \cdot 1$ & $2 \cdot 0 \mathrm{NS}$ & $2 \cdot 1$ & $1 \cdot 8$ & $2 \cdot 2 \mathrm{NS}$ \\
\hline \multicolumn{10}{|l|}{ Stiffness } \\
\hline Mean $(\mathrm{mm})$ & 45 & 46 & 43 & 43 & 43 & & 38 & 39 & \\
\hline Mean rank & $2 \cdot 0$ & $2 \cdot 1$ & $1.9 \mathrm{NS}$ & $2 \cdot 0$ & 1.9 & $2 \cdot 1 \mathrm{NS}$ & $1 \cdot 6$ & $2 \cdot 3$ & $2 \cdot 2 \mathrm{NS}$ \\
\hline \multicolumn{10}{|c|}{ Results of Friedman two way analysis of variance by ranks are indicated by: NS=not significant; $\mathrm{p}>0 \cdot 05 ;{ }^{* *} \mathrm{p}<0 \cdot 01$} \\
\hline
\end{tabular}


to use since it required approximately three minutes to complete.

Our method of determining the sensitivity of the index to change in clinical state depends upon the presumption that one week's treatment with a nonsteroidal anti-inflammatory drug reduces the severity of enthesitis in AS. Whereas in rheumatoid arthritis drugs are available which may induce reduction in severity of synovitis in the short term ${ }^{7}$ and objective methods of measuring severity of synovitis are available with which these clinical observations can be confirmed, ${ }^{89}$ this is not the case in AS. We recognise the limitations of assuming a drug effect in this way, but in addition to a reduction in enthesis index score there was also a reduction in the levels of pain after drug therapy. Since all of the patients had classical AS and none had clinical evidence of an active peripheral polyarthritis with synovitis, it appears likely that the severity of joint inflammation associated with enthesitis was indeed modified in these patients.

It is interesting that there was no significant interobserver variability in the measurement of pain and stiffness by VAS in this study as this indicates high reproducibility. A variety of methods of measuring the severity of pain are available ${ }^{10}$ and are of equal efficacy in the assessment of chronic inflammatory joint disease, though the reproducibility of vertical VAS may be low. ${ }^{11}$ Most subjective methods of assessment of chronic inflammatory joint disease, for example the Ritchie articular index in rheumatoid arthritis, do have significant interobserver variability. ${ }^{12}$ Attempts were made to reduce this source of variation with the EI to a minimum. and it was only significant at the first visit. It might be possible to use other approaches to reduce this still further, for example, by aiming to standardise the amount of pressure applied by each observer. It is likely, however, that there would still be some observer variability and that sequential measurements of the same patient or measurements made in the context of a study would need to be made by the same observer or by observers who had been standardised to give a low degree of interobserver error. Uncontrollable events, such as observer illness, could still affect the score, however, by affecting the amount of pressure applied. It may be that by combining all the grades of tenderness an index might be produced with less inter- and intraobserver variability, but at this stage it is not known whether the severity or simply the presence of tenderness over the enthesis is important. ${ }^{13}$ It is also possible that analysis of data being collected at present will enable us to refine the index further, but this has not been the case to date.

This index could prove useful in the clinic to record the course of patients with AS and determine the efficacy of different treatment regimens, for example, drugs or physiotherapy. It is possible that an index such as this may be relevant in the diagnosis of mild or early disease, and perhaps long term studies may show some predictive ability of the enthesis index to indicate future problems. These possibilities have yet to be explored. The enthesis index could be a useful method of assessing the severity of enthesitis as distinct from synovitis in conditions in which these features are known to exist or may coexist, for example, the seronegative spondarthritides and the types of arthritis associated with schistosomiasis. ${ }^{14}$ Measures of severity of pain and stiffness would be expected to reflect the presence of synovitis or enthesitis or other factors and hence would not be useful in this respect. The enthesis index, however, used in conjunction with these scales and the Ritchie articular index might distinguish between a natural history of enthesitis and that of synovitis, in which case an attempt could be made to define different mechanisms in pathogenesis in relation to known serological markers of inflammation, such as the acute phase protein response. Studies to explore these possibilities are in progress.

We wish to acknowledge the help and support of the patients and Sister Irene Prescott's nursing team on ward 12, Freeman Hospital.

\section{References}

1 Hart F Dudley. Clinical features and complications. In: Moll $\mathrm{J} \mathrm{M} \mathrm{H,} \mathrm{ed.} \mathrm{Ankylosing} \mathrm{spondylitis.} \mathrm{Edinburgh,} \mathrm{London,}$ Melbourne, New York: Churchill Livingstone, 1980: 52-68.

2 Ball J. Enthesopathy of rheumatoid and ankylosing spondylitis. Ann Rheum Dis 1971: 30: 213-23.

3 Spencer D G. Adams F G. Horton P W. Buchanan W W. Scintiscanning in ankylosing spondylitis. A clinical, radiological and quantitative radio-isotope study. Br J Rheumatol 1979; 6: 423-31.

4 Sturrock R D. Wojtulewski J A. Hart F D. Spondylometry in a normal population and in ankylosing spondylitis. Rheumatol Rehabil 1973: 12: 135-42.

5 Gerster J G. Plantar fasciitis and achilles tendonitis in 150 cases of seronegative spondarthritis. Rheumatol Rehabil 1980; 19: 218-22.

6 Warwick R. Williams P L. eds. Gray's anatomy. 35th ed. London: Longman. 1973: 322-3.

7 Ceulaer K de. Dick W C. Clinical evaluation of antirheumatic drugs. In: Kelly W N. Harris E D. Ruddy S. Sledge C B Textbook of rheumatology. Philadelphia, London, Toronto: Saunders, 1981: 729-39.

8 Bacon P A. Collins A J. Ring F J. Cosh J A. Thermography in the assessment of inflammatory arthritis. In: Jayson M I V. ed. Diagnosis and management. Clinics in rheumatic diseases. Vol 2. 1. London: Saunders, 1976: 51-9.

9 Dick W C. Grennan D M. Radioisotopes in the study of normal and inflamed joints. In: Jayson M I V, ed. Diagnosis and management. Clinics in rheumatic diseases. Vol 2. 1 . London: Saunders. 1976: 67-76. 
10 Downie W W, Leatham P A, Rind V M, Wright V, Branco J A, Anderson J A. Studies with pain rating scales. Ann Rheum Dis 1978; 37: 378-438.

11 Dixon J S, Bird H A. Reproducibility along a $10 \mathrm{~cm}$ vertical visual analogue scale. Ann Rheum Dis 1981; 40: 87-9.

12 Ritchie D, Boyle J A, McInnes J M, et al. Clinical studies with an articular index for the assessment of joint tenderness in patients with rheumatoid arthritis. $Q J$ Med 1968: 393-406. (New Series XXXVII.)
13 Hart L E, Tugwell P, Buchanan W W, Norman G R, Grace E M, Southwell D. Grading of tenderness as a source of interrater error in the Ritchie articular index. J Rheumatol 1985; 12: 716-7.

14 Atkin S L, Kamel M, El-Hady A M A, El-Badawy S A, El-Ghobary A, Dick W C. Clinical and laboratory studies on patients with schistome mansoni infection. $Q J$ Med 1986; 59: 479-87. 